POS PROCEEDINGS

\title{
CP-odd Higgs boson production in two-photon processes
}

\author{
Ken Sasaki \\ Dept. of Physics, Faculty of Engineering, Yokohama National University \\ Yokohama 240-8501, Japan \\ E-mail: sasaki@ynu.ac.jp \\ Tsuneo Uematsu* \\ Institute for Liberal Arts and Sciences, Kyoto University, Yoshida, Kyoto 606-8501, Japan \\ and Maskawa Institute, Kyoto Sangyo University, Kyoto 603-8555,Japan \\ E-mail: uematsu@scphys.kyoto-u.ac.jp
}

\begin{abstract}
We discuss the CP-odd Higgs boson production through the two-photon processes in $e \gamma$ collisions. First we briefly review the Standard Model Higgs boson production in $e \gamma$ collisions, with special attention to transition form factor and differential production cross section. We then study the production of the CP-odd Higgs boson $A^{0}$ which appears in the extended Higgs sector such as the Minimal Supersymmetric Standard Model (MSSM) or in the Two-Higgs Doublet Models (2HDM). The electroweak one-loop contributions to the scattering amplitude for $e \gamma \rightarrow e A^{0}$ as well as the transition form factor are calculated and expressed in an analytical form. We found that one-loop contribution only comes from top-quark loops. There are no contributions from Wboson loop nor from stop loop. Numerical analysis for the production cross section is presented. It turns out that the $\gamma^{*} \gamma$-fusion is far more dominant over the $Z^{*} \gamma$-fusion.
\end{abstract}

13th International Symposium on Radiative Corrections (Applications of Quantum Field Theory to Phenomenology)

25-29 September, 2017

St. Gilgen, Austria

\footnotetext{
* Speaker.
} 


\section{Introduction}

The Higgs boson with mass about $125 \mathrm{GeV}$ was discovered by ATLAS and CMS at LHC [1] and its spin, parity and couplings were examined [2]. Now it would be intriguing to study its nature in $e^{+} e^{-}$collisions provided by such as linear collider [3]. Along with $e^{+} e^{-}$collider, other options such as $e^{-} e^{-}, e^{-} \gamma$ and $\gamma \gamma$ colliders have also been discussed. See Refs. [4]-[8] and the references therein. We first summarize what we have studied in $[9,10]$ about standard model (SM) Higgs boson $H$ production through two-photon processes in $e \gamma$ collisions where the scattered electron detected in the final state (single tagging) shown in Fig.1. We calculated the so-

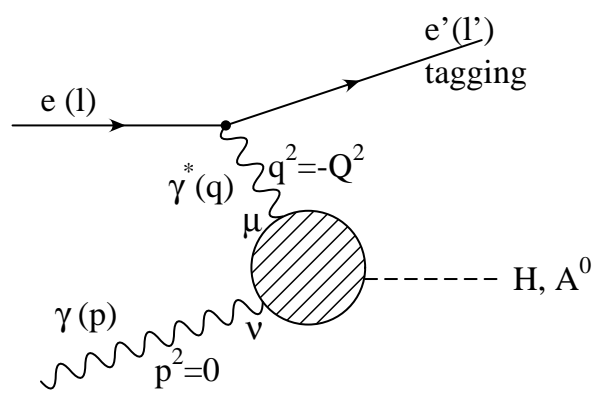

Figure 1: SM Higgs or CP-odd Higgs boson production in $e \gamma$ collision

called "transition form factor" as well as the production cross section, especially their $Q^{2}$ (virtual photon mass squared) dependence. It turned out at one-loop order the $W$-boson loop gives dominant contribution compared to the top-quark loop contribution.

We then extend our argument to CP-odd Higgs boson production in two-photon process of $e \gamma$ collisions [11]. In contrast to SM Higgs boson $H$, CP-odd Higgs $A^{0}$ has much simpler structure in transition form factor. We found that one-loop contribution only comes from top-quark loops. There are no contributions from W-boson loop nor from stop loop. Numerical analysis for the production cross section is presented. It turns out that the $\gamma^{*} \gamma$-fusion is far more dominant over the $Z^{*} \gamma$-fusion.

\section{SM Higgs production in $e \gamma$ collision}

The scattering amplitude for $e(l)+\gamma(p) \rightarrow e^{\prime}\left(l^{\prime}\right)+H(q+p)$ shown in Fig.2 is given by

$$
\left\langle e^{\prime} H|T| e \gamma\right\rangle=\bar{u}\left(l^{\prime}\right)\left(-i e \gamma^{\mu}\right) u(l) \frac{-i}{q^{2}+i \varepsilon} A_{\mu \nu} \varepsilon^{v}(p)
$$

where $\varepsilon^{v}(p)$ is the polarization vector of the incident real photon. Here we have introduced the tensor $A_{\mu v}$ which can be decomposed due to gauge invariance as

$$
A_{\mu v}(q, p)=\left(g_{\mu v}(q \cdot p)-p_{\mu} q_{v}\right) S_{1}\left(m^{2}, Q^{2}, m_{H}^{2}\right)+\left(q_{\mu} p_{v}-\frac{q^{2}}{q \cdot p} p_{\mu} p_{v}\right) S_{2}\left(m^{2}, Q^{2}, m_{H}^{2}\right)
$$

where $q^{2}=-Q^{2}<0, p^{2}=0$ and $(q+p)^{2}=p_{H}^{2}=m_{H}^{2}$. We denote collectively the mass of the intermediate particle in the loop by $m$. Since $p^{v} \varepsilon_{v}(p)=0$, only $S_{1}$ is relevant for the scattering 


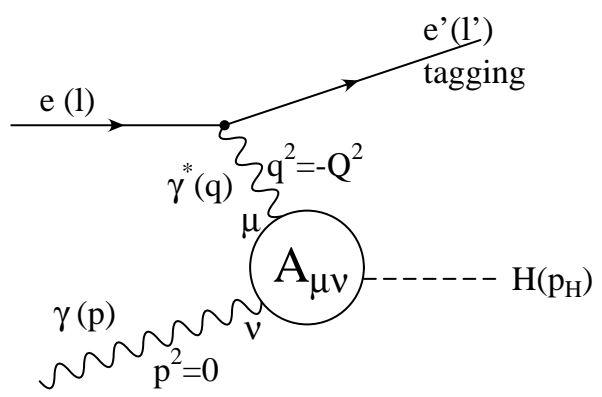

Figure 2: The transition amplitude for Higgs production via virtual and real photon fusion.

amplitude. We define the "transition form factor" $F_{i}\left(m^{2}, Q^{2}, m_{H}^{2}\right)$ by

$$
F_{i}\left(m^{2}, Q^{2}, m_{H}^{2}\right)=S_{1}\left(m^{2}, Q^{2}, m_{H}^{2}\right) /\left(\frac{g e^{2}}{(4 \pi)^{2}} \frac{1}{m_{W}}\right)
$$

where $i=1 / 2,1$ for a fermion-loop $F_{1 / 2}$ and for the W-boson loop $F_{1}$, respectively. $e$ and $g$ are the electromagnetic and weak gauge couplings, respectively, and $m_{W}$ is the $W$ boson mass.

The total transition form factor is given by

$$
F_{\text {total }}\left(Q^{2}, m_{H}^{2}\right)=\sum_{f} N_{c} e_{f}^{2} F_{1 / 2}\left(m_{f}^{2}, Q^{2}, m_{H}^{2}\right)+F_{1}\left(m_{W}^{2}, Q^{2}, m_{H}^{2}\right)
$$

where $N_{c}$ is the number of the colors ( 1 for leptons and 3 for quarks) and $e_{f}$ is the electric charge of the fermion $f$ in the unit of proton charge. Evaluating the production cross section from Eq.(2.4), we found that at the one-loop level the W-loop contribution dominates over top-quark loop [9, 10].

\section{MSSM/2HDM and $A^{0}$ production}

We now consider a minimal extension of the Higgs sector of the Standard Model (SM). Here we investigate the Two-Higgs Doublet Model (2HDM) for the type-II case which includes the MSSM as a special case [12]. We denote the two $\mathrm{SU}(2)_{L}$ doublets $H_{1}, H_{2}$, with weak hypercharge $Y=-1$ and $Y=1$, respectively, by the 4 complex scalar fields, $\phi_{1}^{0}, \phi_{1}^{-}, \phi_{2}^{+}, \phi_{2}^{0}$ as follows:

$$
H_{1}=\left(\begin{array}{c}
H_{1}^{1} \\
H_{1}^{2}
\end{array}\right)=\left(\begin{array}{c}
\phi_{1}^{0 *} \\
-\phi_{1}^{-}
\end{array}\right), \quad H_{2}=\left(\begin{array}{c}
H_{2}^{1} \\
H_{2}^{2}
\end{array}\right)=\left(\begin{array}{c}
\phi_{2}^{+} \\
\phi_{2}^{0}
\end{array}\right)
$$

where, in the type II model, $H_{1}\left(H_{2}\right)$ only couples to the down-type (up-type) quarks and leptons. They acquire the following vacuum expectation values after the spontaneous symmetry breaking:

$$
\left\langle H_{1}\right\rangle=\left(\begin{array}{c}
v_{1} \\
0
\end{array}\right), \quad\left\langle H_{1}\right\rangle=\left(\begin{array}{c}
0 \\
v_{2}
\end{array}\right), \quad \tan \beta=v_{2} / v_{1}
$$

Then 3 degrees of freedom out of 8 consisting of the 4 complex scalar fields are absorbed by the longitudinal components of $W^{ \pm}, Z$, and the remaining 5 physical degrees of freedom become the following two charged and three neutral physical Higgs bosons:

$$
\text { Charged } H^{+}, H^{-} ; \quad \text { CP-even } \quad h^{0}, H^{0} ; \quad \text { CP-odd } \quad A^{0}
$$


Here we are particularly interested in the CP-odd Higgs boson $A^{0}$, and investigate its transition form factor and the production cross section in the $\gamma^{*} \gamma$-fusion process.

We now enumerate some characteristics of the coupling of $A^{0}$ for the type II case:

1) In contrast to the CP-even Higgs bosons $h^{0}$ and $H^{0}, A^{0}$ does not couple to $W^{+} W^{-}$and $Z Z$ pairs at tree level. Hence $W$-boson and $Z$-boson one-loop diagrams do not contribute to the $A^{0}$ production.

2) $A^{0}$ does not couple to other two physical Higgs bosons in cubic interactions.

3) The couplings of $A^{0}$ to the fermions are proportional to the fermion masses. Therefore, we only consider the top quark for the charged fermion loop diagrams. The $A^{0}$ coupling to the top quark with mass $m_{t}$ is given by $\lambda \gamma_{5}$ with [12]

$$
\lambda=-\frac{g m_{t} \cot \beta}{2 m_{W}} .
$$

Here $g$ and $m_{W}$ are the weak gauge coupling and the weak boson mass, respectively.

4) In the case of MSSM, the trilinear $A^{0}$ coupling to mass-eigenstate squark pairs $\tilde{q}_{i} \tilde{q}_{i}(i=1,2)$ vanishes [12]. Hence, the scalar top-quark (stop) does not contribute to the $A^{0}$ production in er collisions at one-loop level.

\subsection{Scattering Amplitude for $\gamma^{*} \gamma$ Fusion Process}

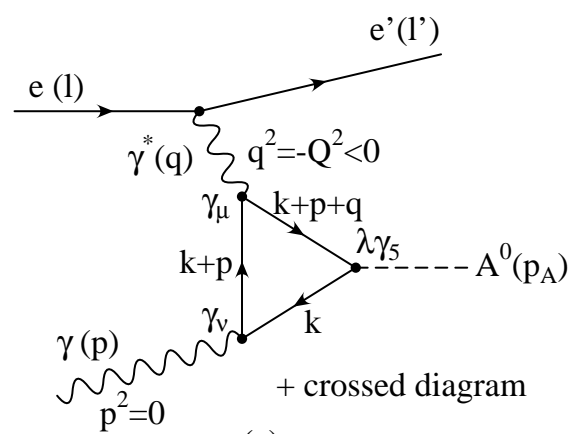

(a)

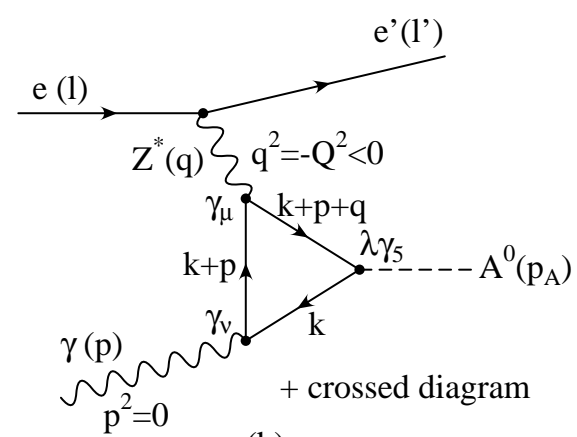

(b)

Figure 3: (a) $\gamma^{*} \gamma$ fusion diagram for $e \gamma \rightarrow e^{\prime} A^{0}$ (b) $Z^{*} \gamma$ fusion diagram for $e \gamma \rightarrow e^{\prime} A^{0}$

We first consider the case of $\gamma^{*} \gamma$ fusion process as shown in Fig.3(a). Since $p$ is the momentum of a real photon, we have $p^{2}=0$ and $p^{v} \varepsilon_{v}(p)=0$, where $\varepsilon_{v}(p)$ is the photon polarization vector. We set virtual photon momentum $q=l-l^{\prime}$. Assuming that electrons are massless so that $l^{2}=l^{\prime 2}=0$, we introduce the following Mandelstam variables:

$$
\begin{aligned}
& q+p=p_{A}, \quad q^{2}=-Q^{2}, \quad p^{2}=0 \\
s= & (l+p)^{2}=2 l \cdot p, \quad t=\left(l-l^{\prime}\right)^{2}=q^{2}=-Q^{2}=-2 l \cdot l^{\prime}, \\
u= & \left(p-l^{\prime}\right)^{2}=-2 l^{\prime} \cdot p=m_{A}^{2}-s-t .
\end{aligned}
$$


where $p_{A}^{2}=m_{A}^{2}$ with $m_{A}$ being the CP-odd Higgs boson mass.

We evaluate the top-loop amplitude for the $\gamma^{*} \gamma$ fusion diagram as shown in Fig.3(a):

The scattering amplitude for $e(l)+\gamma(p) \rightarrow e^{\prime}\left(l^{\prime}\right)+A^{0}\left(p_{A}\right)$ is given by

$$
\left\langle e^{\prime} A|T| e \gamma\right\rangle_{\gamma^{*} \gamma}=\bar{u}_{r^{\prime}}\left(l^{\prime}\right)\left(-i e \gamma_{\mu}\right) u_{r}(l) \frac{-i}{q^{2}+i \varepsilon} A^{\mu v} \varepsilon_{v}\left(p, \lambda_{2}\right)
$$

where $\varepsilon_{v}\left(p, \lambda_{2}\right)$ is the polarization vector of the incident real photon with momentum $p$ and helicity $\lambda_{2}$. The $u_{r}(l)\left(\bar{u}_{r^{\prime}}\left(l^{\prime}\right)\right)$ is the spinor for the initial (scattered) electron with momentum $l\left(l^{\prime}\right)$ and helicity $r\left(r^{\prime}\right)$. The tensor $A_{\mu \nu}$ is given as

$$
A_{\mu v}=-8 i e^{2} m_{t} \lambda \varepsilon_{\mu v \alpha \beta} q^{\alpha} p^{\beta} \int \frac{d^{4} k}{(2 \pi)^{4}} \frac{1}{\left[k^{2}-m_{t}^{2}\right]\left[(k+p)^{2}-m_{t}^{2}\right]\left[(k+p+q)^{2}-m_{t}^{2}\right]} .
$$

\subsection{One-loop integrals}

The above one-loop integral (3.9) is given by the three-point scalar integral by PassarinoVeltman [13]:

$$
\frac{1}{(2 \pi)^{4}} \int d^{4} k \frac{1}{\left[k^{2}-m_{1}^{2}\right]\left[\left(k+p_{2}\right)^{2}-m_{2}^{2}\right]\left[\left(k+p_{1}+p_{2}\right)^{2}-m_{3}^{2}\right]}=\frac{i \pi^{2}}{(2 \pi)^{4}} C_{0}\left(p_{1}^{2}, p_{2}^{2}, p_{3}^{2}, m_{1}^{2}, m_{2}^{2}, m_{3}^{2}\right)
$$

In our present case of $e$ and real $\gamma$ collision we have

$$
C_{0}\left(-Q^{2}, 0, m_{A}^{2}, m_{t}^{2}, m_{t}^{2}, m_{t}^{2}\right)=-\frac{1}{Q^{2}+m_{A}^{2}}\left\{\frac{1}{2} g(\rho)+2 f(\tau)\right\}
$$

where the dimesionless variables $\tau$ and $\rho$ are defined as

$$
\tau \equiv \frac{4 m_{t}^{2}}{m_{A}^{2}}, \quad \rho \equiv \frac{Q^{2}}{4 m_{t}^{2}}
$$

and the two functions $f(\tau)$ and $g(\rho)$ we have introduced are given by

$$
\begin{array}{rlrl}
f(\tau) & =\left[\sin ^{-1} \sqrt{\frac{1}{\tau}}\right]^{2} & \tau \geq 1 \\
& =-\frac{1}{4}\left[\log \frac{1+\sqrt{1-\tau}}{1-\sqrt{1-\tau}}-i \pi\right]^{2} & & \tau<1 \\
g(\rho) & =\left[\log \frac{\sqrt{\rho+1}+\sqrt{\rho}}{\sqrt{\rho+1}-\sqrt{\rho}}\right]^{2} &
\end{array}
$$

Thus we have [11]

$$
A_{\mu \nu}=\frac{g e^{2}}{(4 \pi)^{2}} \frac{\cot \beta}{2 m_{W}} \frac{\tau}{1+\rho \tau}[g(\rho)+4 f(\tau)] \varepsilon_{\mu v \alpha \beta} q^{\alpha} p^{\beta}
$$

where we have used the relation $\lambda=-g m_{t} \cot \beta / 2 m_{W}$. Similar combinations of functions $f(\tau)$ and $g(\rho)$ as in Eq.(3.11) with the time-like virtual mass, appear in the Higgs decay processes $H \rightarrow \gamma^{*} \gamma$ and $H \rightarrow Z^{*} \gamma$ in Ref.[14] (see also Ref.[12] for on-shell decays, $H \rightarrow \gamma \gamma$ [15] and $H \rightarrow Z \gamma$ ). 


\subsection{Transition Form Factor}

We can define the so-called "Transition Form Factor" as in the case of the standard Higgs boson. Now first we note

$$
A_{\mu v}(q, p) \equiv \tilde{S}\left(m_{t}^{2}, Q^{2}, m_{A}^{2}\right) \varepsilon_{\mu v \alpha \beta} q^{\alpha} p^{\beta}
$$

where we now introduce the transition form factor $\tilde{F}$ as

$$
\begin{aligned}
\tilde{F}\left(m_{t}^{2}, Q^{2}, m_{A}^{2}\right) & \equiv \tilde{S}_{1}\left(m_{t}^{2}, Q^{2}, m_{A}^{2}\right) /\left(\frac{g e^{2}}{(4 \pi)^{2}} \frac{1}{m_{W}}\right) \\
& =-\frac{1}{2} \frac{\tau}{1+\rho \tau}[g(\rho)+4 f(\tau)]=4 m_{t}^{2} C_{0}
\end{aligned}
$$

Since $\tau=\left(2 m_{t} / m_{A}\right)^{2}$ we have the following two cases depending on the mass of $A^{0}$.

For $m_{A}<2 m_{t}$ i.e. $\tau>1$ we have $f(\tau)$ given by Eq.(3.13) which is a real function. While for $m_{A}>2 m_{t}$ i.e. $\tau<1$ we have $f(\tau)$ given by Eq.(3.14) which is a complex function. Therefore, the former is real while the latter becomes complex.

We also introduce the total transition form factor $\tilde{F}_{\text {total }}$ which includes the all the flavors of the quark-loop, but dominated by top loop.

$$
\tilde{F}_{\text {total }}\left(Q^{2}\right)=\sum_{f} N_{c} q_{f}^{2} \tilde{F}\left(\rho_{f}, \tau_{f}\right) \simeq 3 \cdot\left(\frac{2}{3}\right)^{2} \tilde{F}\left(m_{t}^{2}, Q^{2}, m_{A}^{2}\right)
$$

We consider the two cases: (a) $m_{A}=300 \mathrm{GeV}$ and (b) $m_{A}=400 \mathrm{GeV}$ shown below (Fig.4(a),(b)).

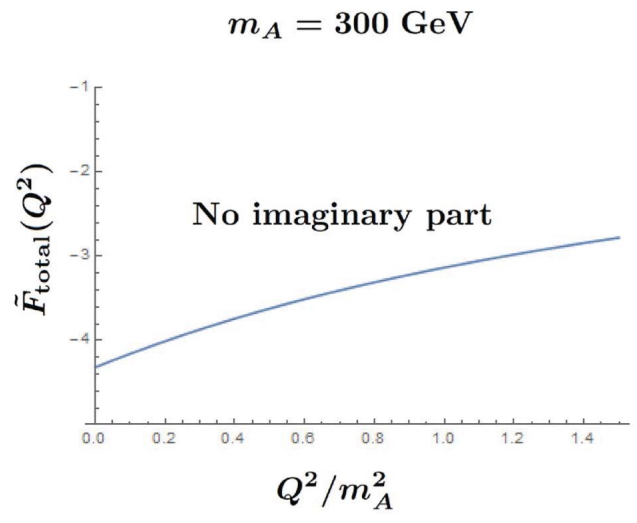

(a)

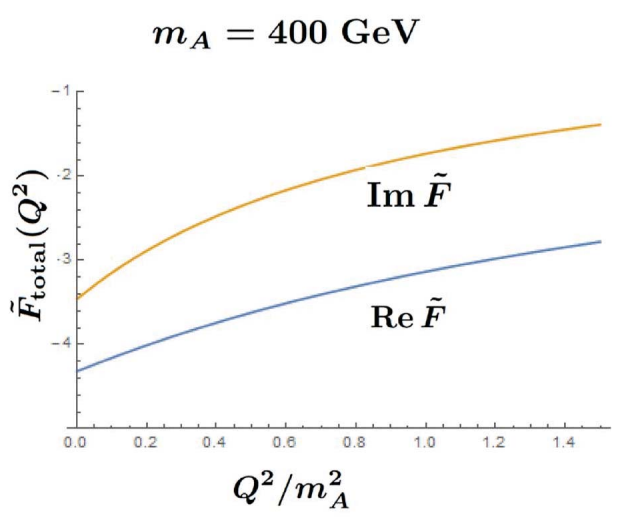

(b)

Figure 4: Transition Form Factor for the mass (a) $m_{A}=300 \mathrm{GeV} \quad$ (b) $m_{A}=400 \mathrm{GeV}$ 


\subsection{Differential cross section}

The differential cross section for the CP-odd Higgs production via $\gamma^{*} \gamma$ fusion in $e \gamma \rightarrow e A^{0}$ is given by

$$
\frac{d \sigma\left(\gamma^{*} \gamma\right)}{d Q^{2}}=\frac{\alpha_{\mathrm{em}}^{3}}{64 \pi} \frac{g^{2}}{4 \pi} \frac{1}{Q^{2}}\left[1+\frac{u^{2}}{s^{2}}\right] \frac{1}{m_{W}^{2}}\left|\tilde{F}_{\text {total }}\left(Q^{2}\right)\right|^{2}
$$

where $\alpha_{\mathrm{em}}=e^{2} / 4 \pi$. Since $s=2 l \cdot p$ and $u=-2 p \cdot l^{\prime}$ we note $u=m_{A}^{2}+Q^{2}-s$. Hence we obtain

$$
\frac{d \sigma\left(\gamma^{*} \gamma\right)}{d Q^{2}} / \frac{\alpha_{\mathrm{em}}^{3}}{64 \pi} \frac{g^{2}}{4 \pi}=\frac{1}{Q^{2}}\left[1+\frac{\left(m_{A}^{2}+Q^{2}-s\right)^{2}}{s^{2}}\right] \frac{1}{m_{W}^{2}}\left|\frac{4}{3} \tilde{F}\left(m_{t}^{2}, Q^{2}, m_{A}^{2}\right)\right|^{2}
$$

\section{5 $\mathrm{Z}$ boson and real $\gamma$ fusion}

The scattering amplitude for $e(l)+\gamma(p) \rightarrow e^{\prime}\left(l^{\prime}\right)+A^{0}\left(p_{A}\right)$ via $Z^{*} \gamma$ fusion shown in Fig.3(b) is

$$
\left\langle e^{\prime} A|T| e \gamma\right\rangle_{Z^{*} \gamma}=\frac{g}{4 \cos \theta_{W}} \bar{u}_{r^{\prime}}\left(l^{\prime}\right)\left(i \gamma_{\mu}\right)\left(f_{Z e}+\gamma_{5}\right) u_{r}(l) \frac{-i}{q^{2}-m_{Z}^{2}} \widetilde{A}^{\mu v} \varepsilon_{v}\left(p, \lambda_{2}\right)
$$

where

$$
\widetilde{A}_{\mu v}=8 e \frac{g}{4 \cos \theta_{W}} m_{t} \lambda f_{Z t} \frac{1}{16 \pi^{2}} C_{0}\left(-Q^{2}, 0, m_{A}^{2}, m_{t}^{2}, m_{t}^{2}, m_{t}^{2}\right) \varepsilon_{\mu v \alpha \beta} q^{\alpha} p^{\beta}
$$

and $e-e-Z$ coupling $f_{Z e}$ and $t-t-Z$ coupling $f_{Z t}$ are given by

$$
f_{Z e}=\left(-1+4 \sin ^{2} \theta_{W}\right), \quad f_{Z t}=\left(1-\frac{8}{3} \sin ^{2} \theta_{W}\right)
$$

Note that the scattering amplitudes both from $\gamma^{*} \gamma$-fusion and $Z^{*} \gamma$-fuison should be added up. When taking absolute square of the amplitude we get an interference term, which turns out to be positive, in addition to the $\gamma^{*} \gamma$ - as well as $Z^{*} \gamma$-fusion terms as follows:

$$
\frac{d \sigma}{d Q^{2}}(\text { total })=\frac{d \sigma}{d Q^{2}}\left(\gamma^{*} \gamma-\text { fusion }\right)+\frac{d \sigma}{d Q^{2}}\left(Z^{*} \gamma-\text { fusion }\right)+\frac{d \sigma}{d Q^{2}}(\text { Interference })
$$

\section{Numerical Analysis}

\section{1 production cross section}

First let us note that the contribution from the $\gamma^{*} \gamma$ fusion is far more dominant over that from $Z^{*} \gamma$-fusion as well as the interference term. We have shown the differential cross sections for the three process in Fig.5 in the case of $\sqrt{s}=500 \mathrm{GeV}, m_{t}=173 \mathrm{GeV}, m_{A}=400 \mathrm{GeV}, \cot \beta=1$. (In fact, the cross sections are proportional to $\cot ^{2} \beta$.) We observe that at $Q^{2}=1000(5000) \mathrm{GeV}^{2}$ the ratio of $d \sigma / d Q^{2}\left(Z^{*} \gamma\right)$ to $d \sigma / d Q^{2}\left(\gamma^{*} \gamma\right)$ is $4.3 \times 10^{-6}\left(5.2 \times 10^{-5}\right)$ and $d \sigma / d Q^{2}$ (Interference) to $d \sigma / d Q^{2}\left(\gamma^{*} \gamma\right)$ is $4.1 \times 10^{-3}\left(1.4 \times 10^{-2}\right)$. Thus the $Z^{*}$-boson exchange reaction does not actually affect the $\gamma^{*} \gamma$ exchange process (Fig.3). This means that the transition form factor makes sense for the $A^{0}$ production in $e \gamma$ collision.

Now we shall focus on the $\gamma^{*} \gamma$ fusion process based on the formula for the production cross section Eq.(3.20). In Fig.6 we have plotted the differential production cross section of $A^{0}$ with 


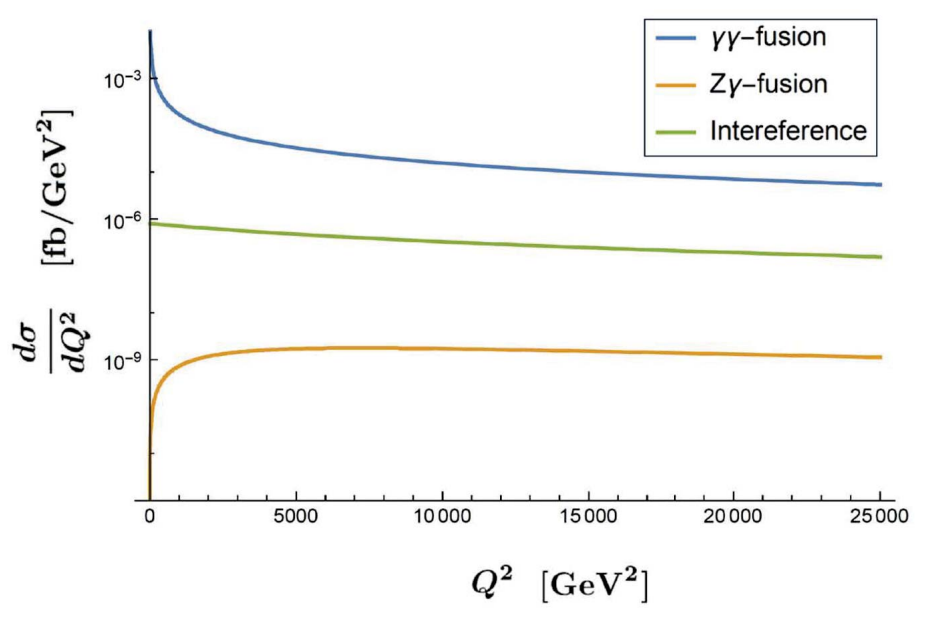

Figure 5: Comparison of the contributions from 3 processes: $\gamma^{*} \gamma$-fusion, $Z^{*} \gamma$-fusion and Interference for $\sqrt{s}=500 \mathrm{GeV}, m_{t}=173 \mathrm{GeV}, \cot \beta=1, m_{A}=400 \mathrm{GeV}$.

mass $m_{A}=200,300,400 \mathrm{GeV}$, for $\sqrt{s}=500 \mathrm{GeV}$ and $m_{t}=173 \mathrm{GeV}$. We find that for this kinematical region the production cross section for $A^{0}$ increases as $m_{A}$ gets larger which looks somewhat unexpected result.

We can examine this behaviour in more detail by computing the differential cross section for fixed $Q^{2}$, which we take to $(100)^{2} \mathrm{GeV}^{2}$.

\subsection{The $A^{0}$ mass dependence of the production cross section}

We have plotted the $A^{0}$ mass dependence of the differential cross section $d \sigma / d Q^{2}$ for $Q^{2}=$ $(100)^{2} \mathrm{GeV}^{2}$ in Fig.7(a) as well as that for the total cross section $\sigma_{\text {total }}$ in Fig.7(b). As $m_{A}$ varies across the $t \bar{t}$ threshold $2 m_{t} \approx 346 \mathrm{GeV}$, the differential cross section $d \sigma / d Q^{2}$ increases in the region $m_{A}<2 m_{t}$, and it turns to decrease when $m_{A}$ goes beyond $2 m_{t}$.

In both cases, we see the strong kink structure corresponding to the threshold effect at $m_{A}=$ $2 m_{t} \approx 346 \mathrm{GeV}$.

\section{Conclusion}

In this talk we have investigated the possible production of the CP-odd Higgs boson $A^{0}$ which would appear in the 2HDM/MSSM through $e \gamma$ collisions. In contrast to the SM Higgs boson $H$ as well as to the CP-even Higgs boson $h^{0}$ and $H^{0}$, the $A^{0}$ does not couple to $W^{+} W^{-}$and $Z Z$ pairs because of the CP-odd nature. Hence at one-loop order $W^{ \pm}$bosons do not contribute to triangle diagrams for the $A^{0}$ production, and only top-quark one-loop triangle diagram is relevant. There is no scalar top-quark (stop) contribution. The transition form factor shows much simpler structure.

When the mass of the $A^{0}$ boson, $m_{A}$ is smaller than $2 m_{t}$ the transition form factor is a real function of $Q^{2}$, while if $m_{A}$ is larger than $2 m_{t}$, the transition form factor becomes complex. The 


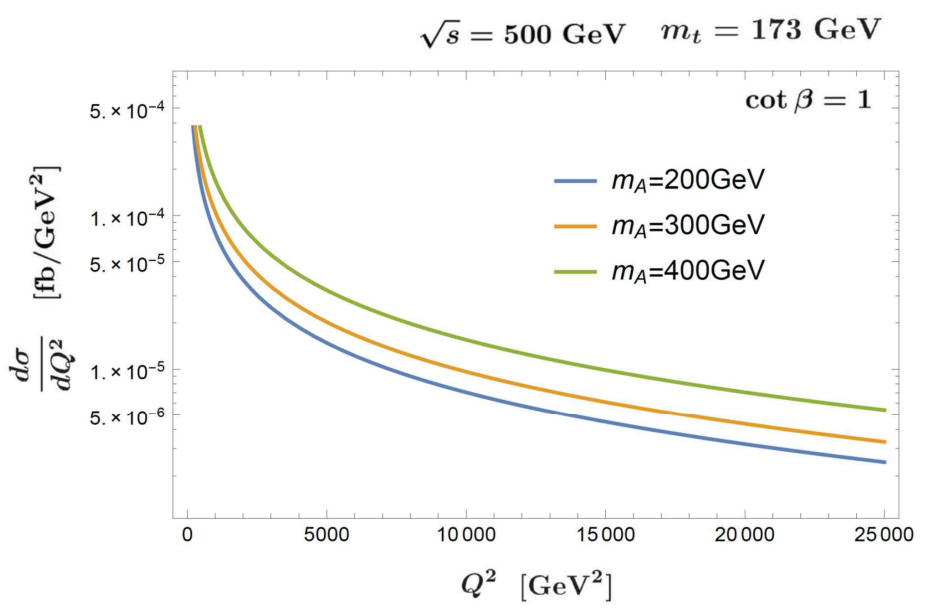

Figure 6: Differential cross section for the production of CP-odd Higgs boson $A^{0}$ with mass $m_{A}=$ 200, 300, $400 \mathrm{GeV}$.

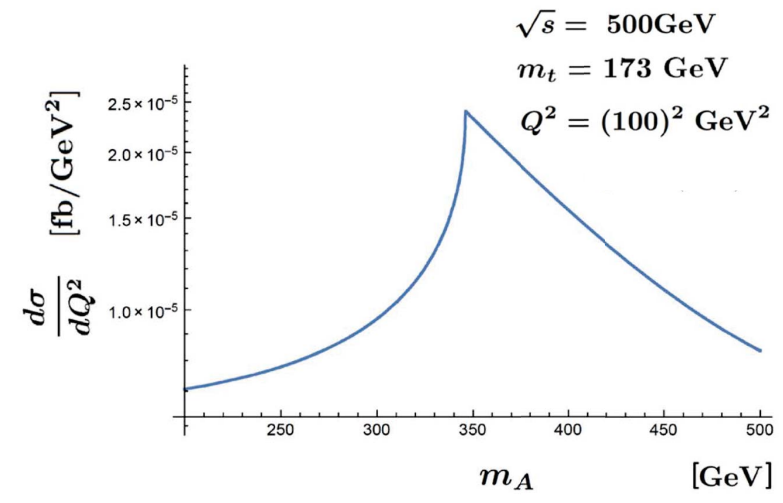

(a)

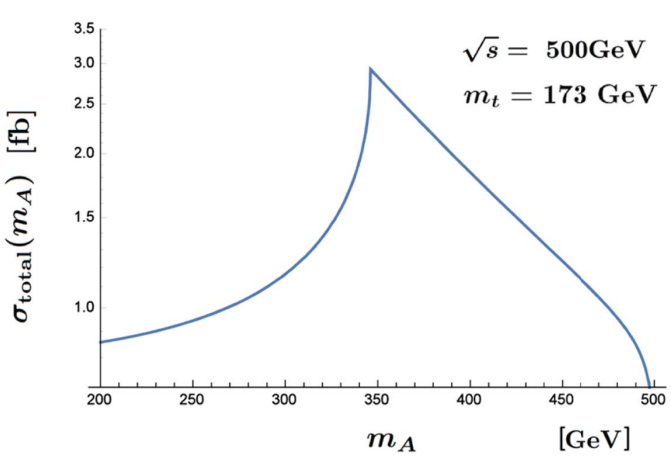

(b)

Figure 7: (a) The $A^{0}$ mass dependence of the differential cross section for the CP-odd Higgs production with $Q^{2}=(100)^{2} \mathrm{GeV}^{2}$ (b) The $A^{0}$ mass dependence of the total cross section for the CP-odd Higgs production.

production cross section of CP-odd Higgs boson is given by the absolute square of the transition form factor together with some kinematical factors.

For a fixed value of $m_{A}$, the differential production cross section shows a decreasing function of $Q^{2}$. On the other hand, if we fix $Q^{2}$ and vary the mass of $A^{0}$, it increases as $m_{A}$ for $m_{A}<2 m_{t}$ and decreases for $m_{A}>2 m_{t}$. This feature is common with the total cross section.

In the $e \gamma$ scattering, the contribution from the $\gamma^{*} \gamma$ fusion is far more dominant over that from $Z^{*} \gamma$ fusion. Thus actually we only have to consider the photon- exchange diagram, and it makes sense to talk about the transition form factor of $A^{0}$. 


\section{Acknowledgments}

We would like to thank the organizers of the RADCOR 2017 for the hospitality at such a well-organized and stimulating symposium.

\section{References}

[1] ATLAS Collaboration, Phys. Lett. B716, 1 (2012); CMS Collaboration, Phys. Lett. B716, 30 (2012).

[2] ATLAS Collaboration, Phys. Lett. B726, 88 (2013); Phys. Lett. B726, 120 (2013); CMS

Collaboration, Phys. Rev. Lett. 110, 081803 (2013).

[3] http://www.linearcollider.org.

[4] A. De Roeck, "Physics at a $\gamma^{*} \gamma, e \gamma$ and $e^{-} e^{-}$Option for a Linear Collider", arXiv:hep-ph/0311138 (2003).

[5] V. I. Telnov, Nucl. Instrum. Meth. A455, 63 (2000) [hep-ex/0001029]; B. Badelek et al., Int. J. Mod. Phys. A19, 5097 (2004) [hep-ex/0108012]; M. M. Velasco et al., eConf C010630, E3005 (2001) [hep-ex/0111055].

[6] M. Melles, W. J. Stirling and V. A. Khoze, Phys. Rev. D61, 054015 (2000); M. Melles, Nucl. Phys. Proc. Suppl. 82, 379 (2000); G. Jikia and S. Soldner-Rembold, Nucl. Phys. Proc. Suppl. 82, 373 (2000); M. M. Mühlleitner, M. Krämer, M. Spira and P. M. Zerwas, Phys. Lett. B508 (2001) 311; M. M. Mühlleitner, Acta Phys. Polon. B37 1127 (2006), [hep-ph/0512232]; D. M. Asner, J. B. Gronberg, and J. F. Gunion, Phys. Rev. D67, 035009 (2003); S. J. Brodsky, Int. J. Mod. Phys. A20, 7306 (2005); P. Niezurawski, eConf C050318, 0503 (2005).

[7] S. A. Bogacz et al., "SAPPHiRE: a Small $\gamma \gamma$ Higgs Factory”, arXiv:1208.2827 [physics.acc-ph] (2012).

[8] I. F. Ginzburg and M. Krawczyk, "Testing Higgs Physics at the Photon Collider", arXiv:1310.5881 [hep-ph] (2013); I. F. Ginzburg and M. V. Vychugin, Physics of Atomic Nuclei, 67, (2004) 281.

[9] N. Watanabe, Y. Kurihara, K. Sasaki and T. Uematsu, Phys. Lett. B728, 202 (2014); PoS (RADCOR 2013) 050; PoS (RADCOR 2013) 053; PoS (QFTHEP 2013) 040.

[10] N. Watanabe, Y. Kurihara, T. Uematsu,and K. Sasaki, Phys. Rev. D90, 033015 (2014).

[11] K. Sasaki and T. Uematsu, arXiv:1712.00197 [hep-ph] (2017).

[12] J. F. Gunion, H. E. Haber, G. Kane and S. Dawason, “The Higgs Hunter's Guide”(Addison-Wesley, 1990).

[13] G. Passarino and M. Veltman, Nucl. Phys. B160, 151 (1979); G. 't Hooft and M.J.G. Veltman, Nucl. Phys. B153, 365 (1979); G.J. van Oldenborgh and J.A.M. Vermaseren, Z. Physik C46 425 (1990).

[14] J.C. Romao and S. Andringa, Eur. Phys. J. C. 7, 631 (1997).

[15] J. Ellis, M. K. Gaillard and D. V. Nanopoulos, Nucl. Phys. B106, 292 (1976); B. L. Ioffe and V. A. Khoze, Sov. J. Part. Nucl. 9, 50 (1978); M. A. Shifman, A. I. Vainshtein, M. B. Voloshin and V. I. Zakharov, Sov. J. Nucl. Phys. 30, 711 (1979); Phys. ReV. D85, 013015 (2012); T. G. Rizzo, Phys. ReV. D22, (1980) 178; M. B. Gavela, G. Girardi, C. Malleville and P. Sorba, Nucl. Phys. B193,257 (1981); W. J. Marciano, C. Zhang and S. Willenbrock, Phys. ReV. D85, 013002 (2012). 